\title{
DETERMINING THE FERMI SURFACE FROM ARPES INTEGRALS
}

\author{
K. MATHO* \\ Centre de Recherches sur les Très Basses Températures \\ Laboratoire associé à l'Université Joseph Fourier, CNRS \\ B.P. 166, 38042 Grenoble cedex 9 , France
}

\begin{abstract}
ARPES integrals are related to the momentum distribution $n_{k}$. In case of a metal, points $k_{\mathrm{F}}$ on the Fermi surface were identified by: (i) a change of sign in the temperature variation of the ARPES integrals or (ii) maximum slope in its angular variation. These criteria are based on the assumption of particle-hole symmetry in the vicinity of the Fermi edge. Here, we check (i) and (ii) on the level of the momentum distribution, for an electronic structure with most of its incoherent weight below the Fermi edge. Evaluating $n_{k}(T)$ up to $T \approx \Delta^{*}$, a Fermi liquid coherence energy, we find: criterion (i) remains stable, while (ii) deviates from $k_{F} \propto m^{*} T / k_{F}$. Published data on the hole doped $t-J$ model are examined in this light.

PACS numbers: 71.10.Ay, 71.18.+y, 79.60.-i
\end{abstract}

\section{Introduction}

The energy profiles of angle resolved photoemission spectroscopy (ARPES) near the Fermi edge are modeled with the spectrum $f(\omega / T) A(k, \omega)$ of the hole correlation function in the initial state. An ARPES integral (AI) is the area under a profile at fixed emission angles. The relation to the many-body sum rule $n_{k}(T)=\int f(\omega / T) A(k, \omega) \mathrm{d} \omega$ is then evident [1]. With this interpretation, ARPES has become a major source of information on correlated holes in low-dimensional (D) conductors [2]. A high resolution AI was first studied on $\mathrm{TiTe}_{2}$ [2] and on high- $T_{\mathrm{c}}$ cuprates [3]. Criterion (i) was established in [3], assuming particle-hole symmetry (PHS). The method of extracting many-body parameters from the profiles [2] can be refined by using the AI as a check on the position of $k_{\mathrm{F}}$ [4-6].

In order to improve our understanding of the AI, we investigate loci $k(\mathrm{i})$ or $k$ (ii) (see the above criteria) on $n_{k}(T)$, for a highly asymmetric electronic structure. We have in mind a hole doped, large $U$ Hubbard or $t-J$ model, with its chemical potential just below a correlation gap [7,8]. Available data on the $2 \mathrm{D} t-J$ model, obtained by a $1 / T$ expansion [9], show strongly diverging lines $k(\mathrm{i})$ and $k$ (ii) in the Brillouin zone. For a more quantitative understanding of this phenomenon, we start from $T=0$ and proceed with a model independent Fermi liquid (FL) analysis.

*e-mail: matho@polycnrs-gre.fr 


\section{Algebraic Fermi liquid scenario}

The excitation spectrum $A(k, \omega)=\pi^{-1} \Im G\left(k, \omega-\mathrm{i} 0_{+}\right)$is described by a complex self-energy, $\Sigma(k, \omega)=\omega-G^{-1}(k, \omega)$. The generic FL has the "Luttinger property" $[10,11]$ : the many-body eigenvalue $\Sigma(k, 0)$ and the first energy derivative $\Sigma^{\prime}(k, 0)=1-1 / Z_{k}<0$ are real quantities, defined for any $k$. A point "on the Fermi surface" (FS) satisfies $\Sigma\left(k_{\mathrm{F}}, 0\right)=0$. The sign of $\Sigma(k, 0)$ partitions $k$-space into the "occupied" $(-)$ and "inoccupied" $(+)$ volume. The discontinuity in $n_{k}$ has the amplitude $Z$, the quasiparticle energy is $\epsilon_{k}^{*}=Z \Sigma(k, 0)$. In the following, we assume $k$ to be on a short, linear path in a 2D Brillouin zone with just one FS crossing. The initial distance from the FS is $\left(k-k_{\mathrm{F}}\right)=\left(m^{*} / k_{\mathrm{F}}\right) Z \Sigma(k, 0)$, in terms of an effective mass. To model the FS crossing, only this critical $k$-dependence is important. An omitted $k$-label implies $k=k_{\mathrm{F}}$. An ansatz, incorporating the two leading terms, is $[8,12]$

$$
\Sigma(k, \omega)=\Sigma(k, 0)+\omega-\left[Z_{k} / \omega+G_{\text {inc }}(k, \omega)\right]^{-1} .
$$

The non-additive, Fano-like combination of coherent and incoherent weights [8] causes the typical "Breit-Wigner line shape" [1]. Within the algebraic approximation scheme [12, 13], the metallic self-energy (advanced or retarded) has $n$ poles $(n>0)$ at positions $\omega \neq 0$. Briefly, $G_{\text {inc }}(k, \omega)=P_{n-1}(\omega) / P_{n}(\omega)$ is a polynomial with complex coefficients, obeying a causality condition in the open plane: $\operatorname{sgn}(\Im \omega) \operatorname{sgn}\left[\Im G_{\text {inc }}(k, \omega)\right]=-1$ (the Herglotz condition). The norm $Q_{k}=$ $\int A(k, \omega) \mathrm{d} \omega$ can be in the range $1 \geq Q_{k}>Z_{k}$. The function $G_{\text {inc }}$ is constructed, using the properties of the continued fraction expansion [12]. Spectra for the doped Hubbard model, obtained with $n=2$, illustrate the efficiency of the method [13]. Quasiparticle damping depends on $\Sigma^{\prime \prime}\left(k,-\mathrm{i} 0_{+}\right)$or, equivalently [8],

$$
G_{\text {inc }}\left(k,-\mathrm{i} 0_{+}\right)=Z_{k}\left[\mathrm{i}-\tan \left(\psi_{k}\right)\right] / \Delta_{k}^{*} \quad\left(-\pi / 2<\psi_{k}<\pi / 2\right) .
$$

The pole trajectory $\omega_{k 0} \approx \epsilon_{k}^{*}+\mathrm{i}\left(\epsilon_{k}^{*}\right)^{2} / \Delta^{*}$ defines the quadratic damping rate, scaled by the coherence energy. At least one self-energy pole approaches to distance of order $\Delta^{*}$ from the origin. A real part in Eq. (2) is the first term without PHS.

\section{Momentum distribution $n_{k}(T)$}

For a $G(k, \omega)$ with $n+1$ simple poles $\omega_{k \lambda}$ and residues $R_{k \lambda}, n_{k}(T)$ can be evaluated by means of the digamma function $\Psi(z)=\mathrm{d} / \mathrm{d} z \ln \Gamma(z)$,

$$
n_{k} / Q_{k}=\frac{1}{2}+\frac{1}{\pi Q_{k}} \Im \sum_{\lambda=0}^{n} R_{k \lambda} \Psi\left(\frac{1}{2}+\frac{\omega_{k \lambda}+\mathrm{i} \delta}{2 \pi \mathrm{i} T}\right) .
$$

The effect of a finite mean free path, shifting all pole trajectories by $\mathrm{i} \delta$, will be discussed elsewhere. The ratio $n_{k} / Q_{k}$ depends on $Q^{*}=Z_{k} / Q_{k}$. The "vicinity" of the FS is expressed by small $x=Z \Sigma(k, 0) / \Delta_{k}^{*} \propto k-k_{\mathrm{F}}$, low temperature by small $t=T / \Delta_{k}^{*}$. In this limit (Fig. 1), $n_{k} / Q_{k}$ is a universal function of $x, t, Q^{*}, \psi$, and $\delta$, independent of the approximation-level $n$. To study the low $T$ effect due to $f(\omega / T)$, we use $n=1$ and the $T=\delta=0$ trajectories. The incoherent part is $G_{\text {inc }}^{-1}(k, \omega)=G_{\text {inc }}^{-1}(k, 0)+\omega /\left(Q_{k}-Z_{k}\right)[2]$, an angle $\psi_{k}<0$ shifts the center of gravity below the Fermi edge. 


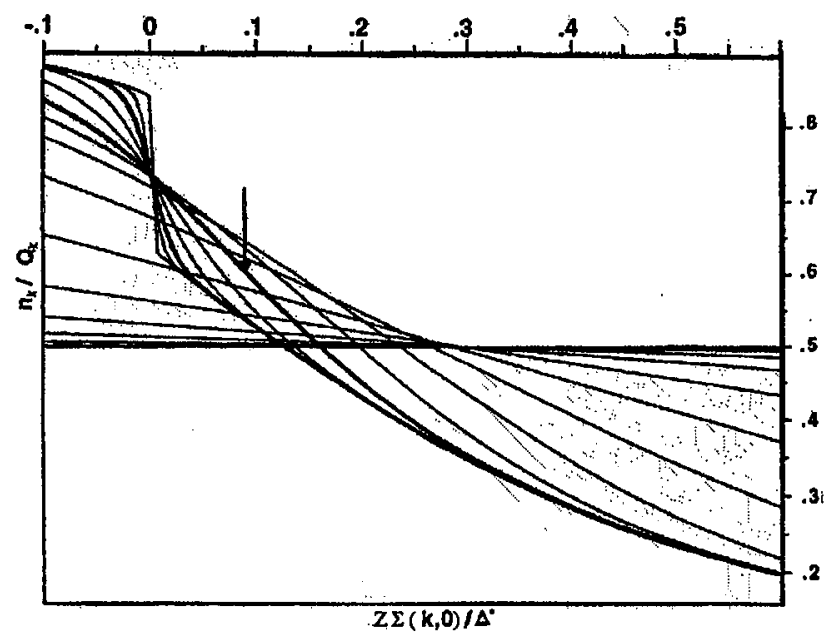

Fig. 1. Scaling plot of the momentum distribution.

\section{Discussion}

We discuss a scaling plot of $n_{k} / Q_{k}$ for $Q^{*}=2 / 9, \psi / \pi=-0.3$ and reduced temperatures $t=0$ and $t=10^{\nu / 3} ;-7 \leq \nu \leq+7$, as function of $x$. Curves $t=0.01$ and $t=0.1$ have slightly bolder lines. In spite of the overall asymmetry, there remains a perfect correlation between the sign of the many-body eigenvalue $\Sigma(k, 0)$ and the sign of the low $t$ variations at constant $k$. The low $t$ "fixed point" is $n_{k_{F}}(0) / Q=\frac{1}{2}-\frac{\psi}{\pi}\left(1-Q^{*}\right)$ [13]. This demonstrates stability of criterion (i).

The vertical arrow on the curve $t=0.1$ indicates the inflexion point, shifting into the unoccupied volume, $x_{i} \propto t$, or $\left(k(\mathrm{ii})-k_{\mathrm{F}}\right) \propto m^{*} T / k_{\mathrm{F}}$. The numerical factor increases or decreases with $\psi$ and $1-Q^{*}$. This shows the instability of (ii).

Further, the scaling plot also reveals non-monotonic $T$-dependence, just outside the FS. The criterion $\mathrm{d} n_{k} / \mathrm{d} T=0$ defines a point $k$ (iii), closer to $k_{\mathrm{F}}$ than $k$ (ii). These conclusions remain unaffected by weak diffusion, $\delta \ll \Delta^{*}$. The self-consistent high- $T$ limit, $n_{k} \equiv m$, required for one-band lattice models with filling factor $m$, is not generally obtained with the low- $T$ spectrum.

The spectral norm of hole doped $t-J$ models, $m<1 / 2$, obeys a Hubbard sum rule: $Q=1-m$. The resulting asymmetric distribution of spectral weight can still be compatible with Luttinger's theorem [7]. The parameters in the scaling plot represent a possible solution at $m=1 / 3$. At this filling, the high- $T$ limit happens to be also correct.

Recent results on $n_{k}(T)$ at $m=0.4$ [9] confirm the tendency of $k$ (ii) to shift into the unoccupied volume. The behavior of the line $k$ (ii) in the Brillouin zone encapsules information on the strong 2D anisotropy of the FL parameters along the FS. A second characteristic line [9] deviates only slightly from a possible Luttinger FS. The authors claim that this line corresponds to criterion (i) [3]. To judge from the scaling plot, it is more likely that they have encountered $k$ (iii). The idea that $k$ (ii) represents the "least biased" criterion for $k_{F}$ has to be abandoned. 
Then, the 1/T-expansion method comes surprisingly close to proving Luttinger's theorem for the $t-J$ model, an objective well worth persuing.

\section{Conclusion}

The applicability of criterion (i) has been extended to arbitrary FL scenarios with quadratic damping, as long as $T \ll \Delta^{*}$. As a consequence, its observation on the AI should not be interpreted as a proof of far reaching PHS, since it is compatible with quite asymmetric electronic structure in the vicinity of the Fermi edge. Only the coherent part, of relative weight $Q^{*}$, i.e. the Landau quasiparticle, has the intrinsic PHS. For models with short-ranged interactions, the 2D non-analytic term $\omega^{2} \ln (\omega)$ is claimed to have negligible influence near the FL fixed point [11]. The present analysis then has a wide range of validity for $D \geq 2$. In particular, it suggests that the normal phase of the $2 \mathrm{D} t-J$ model is a FL. The cuprates have $\Im \Sigma^{\prime}(k, 0) \neq 0$, violating a generic FL property $[12,14]$. On a qualitative level, our conclusions also apply to them.

\section{Acknowledgments}

I thank Roman Micnas for discussions on the Herglotz condition.

\section{References}

[1] L. Hedin, S. Lundquist, Solid State Phys. 23, 1 (1969).

[2] J.W. Allen, G.-H. Gweon, R. Claessen, K. Matho, J. Phys. Chem. Solids 56, 1849 (1995).

[3] Mohit Randeria, Hong Ding, J.-C. Campuzano, A. Bellman, G. Jennings, T. Yokoya, T. Takahashi, H. Katayama-Yoshida, T. Mochiku, K. Kadowaki, Phys. Rev. Lett. 74, 4951 (1995).

[4] R. Claessen, habilitation thesis, Universitaet des Saarlandes, 1997.

[5] G.-H. Gweon, Ph.D. thesis, University of Michigan, Ann Arbor 1999. http://www.umich.edu/gweon/thesis.html.

[6] L. Kipp, K. Rossnagel, J. Bruegmann, C. Solterbeck, T. Strasser, W. Schattke, M. Skibowski, preprint a vailable on http://xxx.lanl.gov, cond-mat/9905400.

[7] K. Matho, J. Magn. Magn. Mater. 108, 170 (1992).

[8] K. Matho, Physica B 199\&200, 382 (1994).

[9] W.O. Putikka, M.U. Luchini, R.R.P. Singh, Phys. Rev. Lett. 81, 2988 (1998).

[10] J.M. Luttinger, Phys. Rev. 119, 1153 (1960).

[11] W. Metzner, C. Castellani, C. Di Castro, Adv. Phys. 47, 317 (1998).

[12] K. Matho, J. Phys. Chem. Solids 56, 1735 (1995).

[13] K. Matho, Mol. Phys. Rep. 17, 141 (1997).

[14] K. Matho, A. Mueller, Physica C 317\&318, 585 (1999). 